\title{
Cardiac involvement in non-human primates infected with the Lyme disease spirochete Borrelia burgdorferi
}

\author{
Diego Cadavid ${ }^{1,2}$, Yunhong Bai ${ }^{1,2}$, Emir Hodzic ${ }^{3}$, Kavitha Narayan ${ }^{1}$, Steven W Barthold ${ }^{3}$ and \\ Andrew R Pachner ${ }^{1}$ \\ ${ }^{1}$ Department of Neurology and Neuroscience; ${ }^{2}$ Center for the Study of Emerging Pathogens, UMDNJ-New \\ Jersey Medical School, Newark, NJ, USA and ${ }^{3}$ Center for Comparative Medicine, University of California at \\ Davis, Davis, CA, USA
}

\begin{abstract}
To investigate cardiac involvement in the non-human primate (NHP) model of Lyme disease, we inoculated 39 adult Macaca mulatta with Borrelia burgdorferi sensu stricto strains N40 (BbN40) by needle $(N=22,14$ immunocompetent (IC), seven permanently immunosuppressed (IS), and four transiently immunosuppressed (TISP)) or by tick-bite $(N=4$, all TISP) or strain 297 (Bb297) by needle $(N=2$ IS), or with $B$. garinii strains $\mathrm{Pbi}$ $(N=4,2$ TISP and 2 IS), $793(N=2$, TISP) or Pli $(N=2$, TISP). Five uninfected NHPs were used as controls. Infection and inflammation was studied in the hearts and the aorta removed at necropsy 2-32 months after inoculation by (1) H\&E and trichrome-staining; (2) immunohistochemistry and digital image analysis; (3) Western blot densitometry; and (4) TaqMan RT-PCR. All NHPs inoculated with BbN40 became infected and showed carditis at necropsy. The predominant cells were T cells, plasma cells, and macrophages. There was increased IgG and IgM in the heart independent of immunosuppression. The B-cell chemokine BLC was significantly increased in IS-NHPs. There was increased deposition of the complement membrane attack complex (MAC) in TISP and IS-NHPs. The spirochetal load was very high in all BbN40-inoculated IS-NHPs but minimal if any in IC or TISP NHPs. Double-immunostaining revealed that many spirochetes in the heart of BbN40-IS NHPs had MAC on their membranes. We conclude that carditis in NHPs infected with B. burgdorferi is frequent and can persist for years but is mild unless they are immunosupressed.
\end{abstract}

Laboratory Investigation (2004) 84, 1439-1450, advance online publication, 27 September 2004; doi:10.1038/labinvest.3700177

Keywords: Borrelia burgdorferi; non-human primates; heart; digital image analysis; plasma cells; BLC; membrane attack complex

Lyme borreliosis is a systemic disease caused by infection with the spirochete Borrelia burgdorferi. ${ }^{1}$ It is currently the most common arthropod-borne disease in the United States, where thousands of cases are reported to the Center for Disease Control every year. ${ }^{2}$ At least three genospecies pathogenic to humans have been characterized: B. burgdorferi sensu stricto, $B$. afzelii, and $B$. garinii. Only $B$. burgdorferi sensu stricto is endemic in North America, while all three genospecies are endemic in Europe. The organs most often affected are the skin, the joints, the heart, and the central and peripheral nervous system.

Correspondence: Dr D Cadavid, MD, Department of Neurology and Neuroscience, UMDNJ-New Jersey Medicine School, 185 South Orange Avenue, MSB H506, Newark, NJ 07103, USA.

E-mail: Cadavidi@umdnj.edu

Received 9 March 2004; revised 13 July 2004; accepted 16 July 2004; published online 27 September 2004
Cardiac manifestations of $B$. burgdorferi infection occur in up to $8 \%$ of patients. ${ }^{1,3}$ Clinically, Lyme carditis is typically characterized by varying degrees of intermittent atrioventricular block occurring within weeks of infection with $B$. burgdorferi, a median of 21 days from the onset of erythema migrans. Temporary cardiac pacing may be required in up to one-third of cases. Myocarditis and/or pericarditis may occur and also mild left ventricular dysfunction that rarely can be fatal. ${ }^{1}$ Cardiomyopathy has been associated with $B$. burgdorferi infection in Europe but not in the United States. ${ }^{4}$

Our laboratory has been studying the pathogenesis of Lyme borreliosis using non-human primates (NHPs) for several years. ${ }^{5-10}$ During infection of immunosuppressed NHPs inoculated with the sensu stricto strain N40 of B. burgdorferi (BbN40), we found that the heart had the most severe injury of all tissues examined and one of the highest spirochetal loads. ${ }^{6}$ The goal of the present study was to 
characterize the full spectrum of Lyme carditis in the NHP model using different strains of $B$. burgdorferi and degrees of immunosuppression during both short- and long-term infection and after syringe or tick-inoculation. The results showed that cardiac inflammation was a consistent finding in all NHPs inoculated with BbN40 but was mild unless the animals were immunosuppressed.

\section{Materials and methods}

\section{Borrelia Strains}

The following Borrelia strains were used for these experiments: B. burgdorferi sensu stricto strains N40 (BbN40) and 297 (Bb297); and B. burgdorferi subspecies garinii strains Pli (BgPli), 793 (Bg793), and Pbi (BgPbi). BbN40 is a North American tickisolate, ${ }^{11} \mathrm{Bb} 297$ is a CSF isolate from a patient in Connecticut. ${ }^{12} \mathrm{BgPbi}$ and $\mathrm{BgPli}$ are CSF isolates from Europe,$^{13}$ and Bg793 is a tick isolate from Europe.

\section{Animals Inoculation and Necropsy}

A total of 39 adult Macaca mulatta were inoculated intradermally with different strains of $B$. burgdorferi sensu stricto or garinii as follows: $\mathrm{BbN40}$ by needle $(N=22)$ or by tick-bite $(N=4) ; \mathrm{Bb} 297$ by needle $(N=2)$; or BgPbi $(N=4)$, Bg793 $(N=2)$ or BgPli $(N=4)$ all by needle. The methods for tick or needle inoculation and for immunosuppression have been reported before ${ }^{5}$ as well as results for infection of all the immunosuppressed animals inoculated with BbN40, Bb297, and garinii strains. The hearts from five NHPs that were uninfected were used as negative controls.

\section{Histology}

Tissues from all organs were processed for histology by routine formalin-fixation and embedding in paraffin or snap-frozen in cryomatrix (Shandon) in isopentane chilled to less than $-140^{\circ} \mathrm{C}$ in liquid nitrogen. Paraffin sections were cut at $5 \mu \mathrm{m}$ and cryomatrix sections at $8 \mu \mathrm{m}$. Inflammation was assessed by examination of hematoxylin and eosin (H\&E) staining. To compare the severity of inflammation in heart, H\&E-stained sections were graded by a masked examiner for the severity of inflammation per $\times 40$ microscopic field as follows: absent inflammation $=0 ; 1-3$ foci $=1+($ minimal $) ; 4-10$ foci $=2+$ (mild); $11-20$ foci $=3+$ (moderate); and more than 20 foci: $4+$ (severe).

\section{Enzyme-Linked Immunosorbent Assay and Immunoblot}

Serum enzyme-linked immunosorbent assay (ELISA) and Western blots were performed as described..$^{5,7,14}$ The strain used for preparation of most Western blots was $B$. burgdorferi sensu stricto strain CB, an isolate from an erythema migrans lesion from Valhalla, NY. ELISA and immunoblots were repeated for the garinii-inoculated NHPs using antigens from $B$. garinii strains.

\section{Immunohistochemistry and Image Analysis}

Immunohistochemistry and digital image analysis were performed as previously described., ${ }^{6,7}$ Antigen retrieval by microwave heating (Dako's target retrival solution, Code No. S1699) or protease digestion (P-6911, Sigma Protease) was used with formalinfixed tissues. Rabbit polyclonal antibody antihuman IgG (Dako’s A0423), IgM (Dako’s A0425), C1q (Dako’s A0136), and CD3 (T cell marker, Dako's A0452), mouse monoclonal antibody anti-P63 (plasma cell marker, Dako's M7077), Ham56 (monocyte/macrophage marker, Dako's M0632), C9 neoepitope (MAC) (Dako's M0777), and goat anti-human BLC/ BCA(R\&D AF801) were used as primary antibody. Recombinant human BLC/BCA peptide (R\&D, 801CX) was used for blocking assays of the anti-BLC antibody to confirm its specificity. Primary antibody for detection of $B$. burgdorferi was hyperimmune serum from a rabbit persistently infected with B. burgdorferi strain N40. ${ }^{15}$ For negative controls, duplicate sections on each glass slide were incubated with affinity-purified nonspecific antibody (Sigma) matched for concentration, species, and isotype. Spleen or lymph node tissues were used as positive controls for markers of inflammation.

The intensity and extent of the immunohistochemical stains were compared by digital image analysis with Image-Pro Plus software 4.1 (Media Cybernetics). For this, a masked examiner (YB) took 4-6 digital images at $\times 40, \times 100$, or $\times 200$ magnification. The mean (s.d.) sum area (in square microns) and sum optical density (in arbitrary units) were determined and compared between groups. The intensity of immunostaining for some markers (CD3, P63, and C1q) was compared manually by semiquantitation of the intensity of the staining as absent (0), mild (1), moderate (2), or severe (3) by a masked examiner.

\section{Immunofluorescence Staining}

C5b-9 (MAC) and B. burgdorferi double immunofluorescence staining was performed with fluorescein isothiocyanate (FITC)-conjugated (Sigma, F0382) anti-rabbit and tetramethyl rhodamine isothiocyanate (Tritc)-conjugated anti-mouse goat polyclonal antibody (Sigma, T6528) at 1/250 dilution. Adobe Photoshop V7.0 software was used to merge single color images.

\section{PCR}

Total RNA was extracted with TRIzole reagent (Life technologies) from $100 \mathrm{mg}$ NHP tissue blocks. The 
reverse transcription (RT) was performed in 20- $\mu$ l reaction volumes. Taqman RT-PCR for the $16 \mathrm{~S}$ rRNA of Borrelia spp. was performed as described. ${ }^{5,7}$ PCRELISA for the OspA or OspB B. burgdorferi genes was performed as described. ${ }^{6}$ All assays were run in triplicate.

\section{Immuno Dot-Blot}

Immuno dot-blot was performed as described. ${ }^{7}$ Protein concentration was determined in the supernatant by the BCA protein assay (Pierce). Dot-blots were prepared by spotting $0.02-0.2 \mu \mathrm{g}$ in duplicate from each protein extract to polyvinylidene difluoride membranes (Millipore). The primary antibodies were rabbit polyclonal anti human IgG (Dako) or IgM at a 1:5000 dilution. The secondary antibody was alkaline-phosphatase-conjugated goat anti-rabbit IgG (Sigma). After incubation in fluorescence substrate ECF (Amersham's RPN5785) for $5 \mathrm{~min}$, the membranes were scanned with the Typhoon 8600 (Amershan Pharmacia Biotech Inc.). Results were analyzed by densitometry using Image-Quant Software and expressed as mean (s.d.).

\section{Statistical Analysis}

For digital image analysis, differences in mean sum density or area were compared for statistical significance using nonparametric tests (Mann-Whitney test) with the SPSS software version 10 . The $P$-values lower than 0.05 were considered significant. For immuno dot-blot, results were compared for significance by Student's $t$-test.

\section{Results}

\section{Animal Infections}

A total of 39 adult NHPs inoculated with different strains of Borrelia burgdorferi were used for these studies. The results of infection of all animals that were immunosuppressed have been published. ${ }^{5}$ Table 1 lists the results of infection of all immunocompetent NHPs inoculated intradermally with $B$. burgdorferi strain N40. None of the NHPs developed erythema migrans and no signs of neurological disease were apparent to care takers. Serial examination of cerebrospinal fluid (CSF) failed to show any evidence of CSF-leukocytosis. Viable spirochetes were identified by tissue culture at necropsy only in immunosuppressed animals.

\section{Antibody Response}

ELISA on necropsy sera showed that all immunocompetent NHPs inoculated with BbN40 developed specific antibody (Table 1). In contrast, only one out of two NHPs inoculated with Bb297 and four out of eight NHPs inoculated with $B$. garinii strains had
Table 1 Infection in immunocompetent NHPs examined by necropsy 2-32 months after intradermal inoculation with $B$. burgdorferi sensu stricto strain N40

\begin{tabular}{lcccc}
\hline NHP ID & $\begin{array}{c}\text { Necropsy } \\
\text { (months) }\end{array}$ & $\alpha B b E L I S A^{\mathrm{a}}$ & $\alpha$ BbIgM WB & $\alpha$ abIgG WB \\
\hline TO26 & 32 & + & - & + \\
TO29 & 31 & + & - & + \\
PAX40 & 20 & + & - & + \\
TO32 & 8 & + & - & + \\
E520 & 6.5 & + & - & + \\
M14 & 6 & + & + & + \\
M15 & 6 & + & + & + \\
E518 & 5 & + & - & + \\
DES9 & 4 & NA & NA & NA \\
E78 & 4 & NA & NA & NA \\
U368 & 4 & NA & NA & NA \\
$23 Z$ & 4 & + & + & + \\
E680 & 4 & + & + & + \\
1616 & 2.5 & + & - & + \\
\hline
\end{tabular}

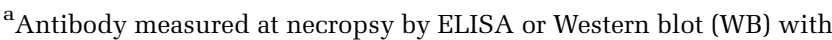
homologous sonicate.

detectable anti $B$. burgdorferi antibody by ELISA and at low titers. ${ }^{5}$ The immunoblot for all inoculated NHPs have been reported.,16,17 Table 1 also summarizes the immunoblot results for the BbN40inoculated immunocompetent NHPs: all that were tested had positive Lyme IgG WB at necropsy and none had positive IgM WB when examined later than 6 months after inoculation. All WB from garinii-inoculated NHPs were negative even when tested with homologous sonicates, ${ }^{5}$ an indication that NHPs are resistant to syringe inoculation of garinii strains of Lyme disease borrelias.

\section{Inflammation in the Heart}

The aorta and the atrium, ventricle and apex of the hearts from all NHPs were examined for the presence of inflammation (carditis) by light microscopy of H\&E-stained paraffin and frozen sections (Figure 1a). The results showed inflammation in at least one cardiac tissue block from all but four of the 39 inoculated NHPs. No carditis was observed in any of the five uninfected controls. To compare the frequency and severity of inflammation, we calculated a mean sum inflammatory score (see Materials and methods) (Table 2). The highest mean sum scores were $0.31,0.30$, and 0.28 for the BbN40inoculated TISP-tick, IS, and TISP-needle NHPs, respectively. The mean sum inflammatory score was also increased in the IC-short-term and long-term groups (0.20 and 0.26$)$ and in the Bg793 garinii group (0.28) (not shown). Trichrome staining of the ventricles of BbN40-inoculated NHPs showed most had increased collagen deposition compared with uninfected controls (Figure 1b). The mean (s.d.) sum density score for collagen per $\times 40$ microscopic field was 2638 (2231), 12327 (11351), 13762 (8176), and 

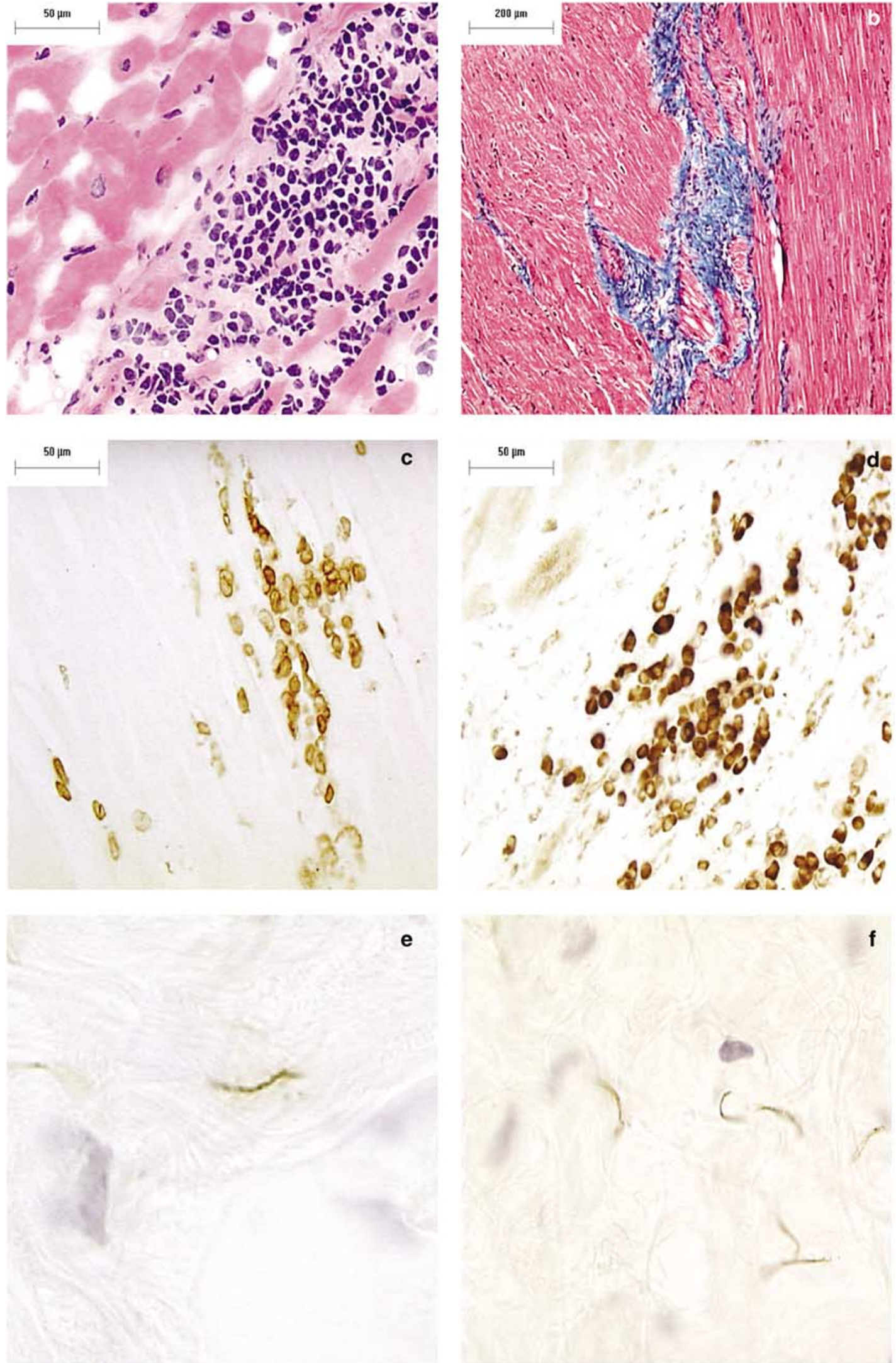

Figure 1 Inflammation in NHP M15 heart inoculated with BbN40 (panel a, H\&E $\times 400$ ); Trichrome staining in 372 IS-NHPs heart (panel b, $\times 100$ ); T cells in IS-NHP 372 heart (panel c, CD3 immunostaining $\times 400$ ); plasma cells in IS-NHP 1614 heart (panel d, P63 immunostaning $\times 400$ ); B. burgdorferi in the aorta (panel e) and atrium (panel f) of IS NHP 571 inoculated with Bb297 (Borrelia immunostaining $\times 1000$ in panel a and $\times 400$ in panel f). 
Table 2 Inflammation by H\&E in biopsy heart from immunocompetent (IC) or transiently (TISP) or permanently (IS) immunosuppressed NHPs inoculated with $B$. burgdorferi N40 by needle or by tick-bite compared with uninfected controls

\begin{tabular}{|c|c|c|c|c|c|c|c|c|c|}
\hline \multirow[t]{2}{*}{ NHP ID } & \multirow[t]{2}{*}{ Strain } & \multirow[t]{2}{*}{ Inoculation } & \multirow{2}{*}{ Immunosuppression } & \multicolumn{4}{|c|}{$H \& E$} & \multicolumn{2}{|c|}{ Mean sum score } \\
\hline & & & & Aorta & Atrium & Ventricle & Apex & $N H P$ & Group \\
\hline TO26 & $\mathrm{BbN} 40$ & Needle & IC-long term & 1 & 1 & NA & NA & $2 / 8^{\mathrm{a}}$ & \\
\hline TO29 & & & $\mathrm{IC}$ & 1 & 1 & 1 & NA & $3 / 12$ & \\
\hline PAX40 & & & IC & 1 & 1 & 1 & NA & $3 / 12$ & \\
\hline TO32 & & & IC & 1 & 2 & 1 & 2 & $6 / 16$ & \\
\hline E520 & & & $\mathrm{IC}$ & 1 & 0 & 1 & NA & $2 / 12$ & $0.26^{\mathrm{b}}$ \\
\hline E518 & $\mathrm{BbN} 40$ & Needle & IC-short term & 2 & 0 & 3 & 0 & $5 / 16$ & \\
\hline DES9 & & & $\mathrm{IC}$ & 1 & 1 & 0 & 1 & $3 / 16$ & \\
\hline E78 & & & IC & 2 & 2 & 2 & 2 & $8 / 16$ & \\
\hline U368 & & & IC & 0 & 0 & 0 & 0 & $0 / 16$ & \\
\hline M14 & & & IC & 0 & 3 & 0 & 1 & $4 / 16$ & \\
\hline M15 & & & IC & 0 & 0 & 1 & 3 & $4 / 16$ & \\
\hline $23 Z$ & & & IC & 1 & 0 & 0 & 0 & $1 / 16$ & \\
\hline E680 & & & IC & 1 & 1 & 0 & 0 & $2 / 16$ & \\
\hline 1616 & & & IC & 3 & 0 & 0 & 0 & $3 / 16$ & 0.20 \\
\hline 652 & $\mathrm{BbN} 40$ & Needle & IS & 0 & 1 & 2 & 2 & $5 / 16$ & \\
\hline 1538 & & & IS & 3 & 1 & 3 & 0 & $7 / 16$ & \\
\hline Z1 & & & IS & 3 & 0 & 0 & 0 & $3 / 16$ & \\
\hline PAX219 & & & IS & 2 & 3 & 1 & 3 & $9 / 16$ & \\
\hline 1614 & & & IS & 2 & 3 & 0 & 0 & $5 / 16$ & \\
\hline 30794 & & & IS & 0 & 0 & 0 & 2 & $2 / 16$ & \\
\hline 372 & & & IS & 0 & 0 & 2 & 1 & $3 / 16$ & 0.30 \\
\hline 099 & $\mathrm{BbN} 40$ & Needle & TISP & 0 & 2 & 3 & 2 & $7 / 16$ & \\
\hline 177 & & & TISP & 0 & 2 & 1 & 0 & $3 / 16$ & \\
\hline 199 & & & TISP & 0 & 2 & 1 & 0 & $3 / 16$ & \\
\hline 383 & & & TISP & 0 & 2 & 2 & 1 & $5 / 16$ & 0.28 \\
\hline 154 & BbN40 & Tick & TISP & 0 & 1 & 1 & 0 & $2 / 16$ & \\
\hline 192 & & & TISP & 0 & 2 & 3 & 1 & $6 / 16$ & \\
\hline 211 & & & TISP & 0 & 3 & 3 & 3 & $9 / 16$ & \\
\hline 242 & & & TISP & 0 & 1 & 1 & 1 & $3 / 16$ & 0.31 \\
\hline M5 & None & None & IC & 0 & 0 & 0 & 0 & $0 / 16$ & \\
\hline M6 & & & IC & 0 & 0 & 0 & 0 & $0 / 16$ & \\
\hline 316 & & & IC & NA & NA & 0 & NA & $0 / 4$ & \\
\hline 650 & & & IC & NA & NA & 0 & NA & $0 / 4$ & \\
\hline 054 & & & IC & NA & NA & 0 & NA & $0 / 4$ & 0 \\
\hline
\end{tabular}

${ }^{a}$ Number of points for inflammation over number of maximum point possible (range 0-4, see Materials and methods for details).

${ }^{\mathrm{b}}$ Sum of all points for inflammation severity in the group divided by number of maximum points possible.

$\mathrm{NA}=$ Not available.

21246 (24 807) for uninfected controls, IS, TISP-tick, and IC-short term NHPs, respectively ( $P$-value $<0.01$ for all groups compared with uninfected controls).

Light microscopic examination indicated that the predominant inflammatory cells were mononuclear cells, many with morphological features of plasma cells. To further characterize the inflammatory infiltrate we did immunostaining for T cells (CD3, Figure 1c), plasma cells (P63, Figure 1d), and macrophages (Ham56) and compared them manually (for T cells and plasma cells, Table 3) or by digital image analysis (for macrophages, Figure 2). The results showed that there were more T cells and plasma cells in the IS group, followed by the shortterm-IC group. The extent of macrophage infiltration was higher in short-term-IC, IS, TISP-tick, and TISPneedle NHPs than in uninfected controls or the long-term-IC group ( $P$-value compared with uninfected controls was $<0.05$ for short-term-IC and $<0.01$ for TISP-needle and TISP-tick).

\section{Spirochetal Localization and Numbers}

To investigate the localization of spirochetes in the heart, we examined tissue sections immunostained with anti-B. burgdorferi specific antibody. Spirochetes were found in the aorta and the heart from BbN40 or Bb297 inoculated IS-NHPs (Figure 1, panels e and f). Some areas had very large numbers of spirochetes, as many as 5-10 per $\times 400$ 
Table 3 Semiquantitation of CD3, P63 and C1q protein expression in the heart of NHPs inoculated with B. burgdorferi sensu stricto strain N40

\begin{tabular}{llcccc}
\hline NHP ID & Inoculation & Immunosuppression & CD3 & P63 & $C 1 q$ \\
\hline E518 & \multirow{2}{*}{ Needle } & IC-short term & $1^{\mathrm{a}}$ & 1 & $0-1$ \\
$23 Z$ & & IC & 1 & 1 & $0-1$ \\
E680 & & IC & 1 & $1-2$ & 2 \\
1616 & IC & $1-2$ & 1 & 0 \\
& & & & & \\
652 & Needle & IS & 2 & 1 & 0 \\
1538 & & IS & $2-3$ & $2-3$ & 0 \\
Z1 & IS & 2 & $2-3$ & 1 \\
PAX219 & & IS & $2-3$ & $2-3$ & 2 \\
1614 & & IS & 2 & 2 & 0 \\
30794 & & IS & 2 & 2 & 1 \\
372 & & IS & 2 & 2 & 1 \\
& & & & & \\
099 & Needle & TISP & 1 & 1 & 0 \\
177 & & TISP & 2 & 1 & 0 \\
199 & & TISP & 2 & 1 & 0 \\
383 & & TISP & 2 & 1 & 0 \\
& & & & & \\
154 & Tick & TISP & 2 & 1 & 0 \\
192 & & TISP & 2 & 1 & 0 \\
211 & & TISP & 2 & 2 & 0 \\
242 & & TISP & 2 & $0-1$ & 0 \\
M5 & None & & & \\
M6 & & IC & 0 & 0 & $0-1$ \\
& & & 0 & 0 & 0
\end{tabular}

${ }^{\mathrm{a}}$ The intensity of the staining as absent (0), mild (1), moderate (2), or frequent (3).

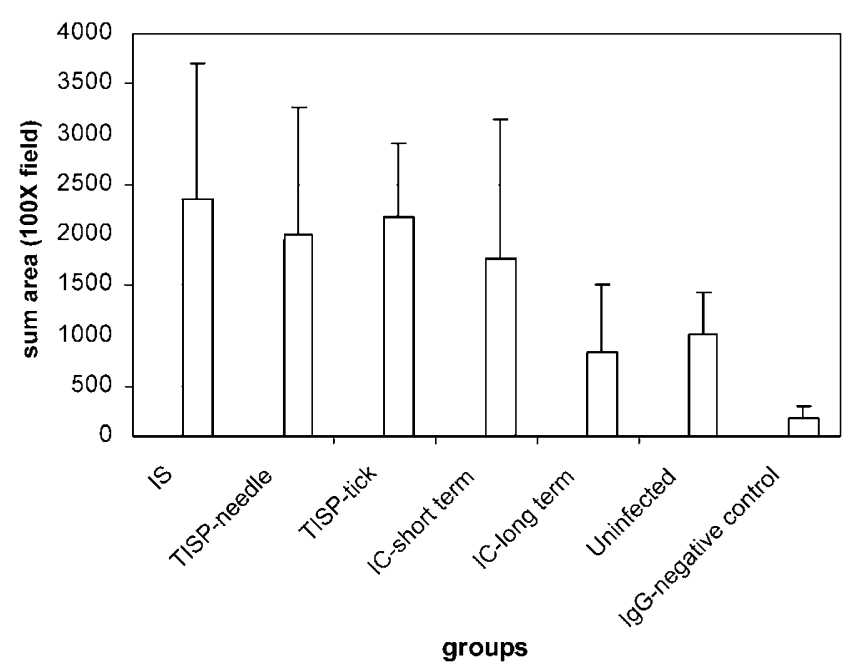

Figure 2 Digital image analysis of immunostaining with antibody anti-Ham 56 (macrophage marker) in the heart from different groups of NHPs inoculated with $B$. burgdorferi by needle or tickbite.

microscopic field (Figure 1f). Their localization was predominantly in connective tissue in the aorta and the heart atrium and ventricle (endocardium, pericardium, and epicardium). In no case, they appeared to be intracellular in macrophages or cardiac myocytes.
To investigate the presence of $B$. burgdorferi in tissues at necropsy, we used OspA or OspB PCRELISA or Borrelia 16S rRNA TaqMan RT-PCR (Table 4). The results showed that in all tissues examined from IC or TISP NHPs the signal was either negative or only weakly positive, with inconsistent results when multiple areas from the same heart were examined (not shown). In contrast, the TaqMan RTPCR detected large numbers of spirochetes in the heart of BbN40-inoculated IS-NHPs (Table 5).

\section{Antibody Deposition}

Plasma cells were common in the heart from all BbN40-inoculated NHPs. Since the production of immunoglobulin is the primary function of plasma cells, we next looked for the presence of antibody in hearts from BbN40-inoculated NHPs. Light microscopic examination revealed extensive deposition of IgG and IgM in the membranes of cardiac myocytes and blood vessels and in the connective tissue throughout the heart and the aorta (not shown). Digital image analysis showed significantly increased deposition of IgG in all BbN40-inoculated NHPs compared with uninfected controls $(P<0.001)$ (Figure 3a). There were also significant differences in IgM deposition (Figure 3b). IS and to a lesser extent TISP-NHPs but not the short-term-IC group had significantly increased IgM deposition compared with uninfected controls $(P<0.01)$.

To confirm if the hearts from IS-NHPs had higher antibody deposition than the TISP groups, we did dot-blot densitometry in whole-protein extracts from ventricles (Table 6). The results confirmed that there was significantly more IgM in IS than in both TISP NHP groups. It also revealed higher IgM in the TISP-needle compared with the TISP-tick and higher IgG in the TISP-needle than in the other two groups.

\section{Complement Deposition}

Immunohistochemistry showed deposition of the first component of the complement cascade (C1q) in the heart from some inoculated NHPs (Figure 4a). The localization was predominantly membrane bound, perivascular, and in collagenous areas. To investigate whether there were differences in the deposition of C1q, we did manual semiquantitation (Table 3). Only some of the short-term-IC and IS NHPs inoculated with BbN40 showed increased C1q deposition by light microscopy. However, dot-blot densitometry showed that the amount of C1q was significantly higher in IS than in any of the two groups of TISP-NHPs (Table 6).

To investigate whether antibody and C1q deposition was associated with deposition of the membrane attack complex (MAC/C5b-9), we did immunohistochemistry with an anti-human MAC primary antibody. The results showed the presence 
Table 4 Infection by PCR in heart from immunocompetent (IC) or transiently (TISP) or permanently (IS) immunosuppressed NHPs inoculated with $B$. burgdorferi $\mathrm{N} 40$ by needle or by tick-bite

\begin{tabular}{|c|c|c|c|c|c|c|c|}
\hline \multirow[t]{2}{*}{ NHP ID } & \multirow[t]{2}{*}{ Strain } & \multirow[t]{2}{*}{ Inoculation } & \multirow[t]{2}{*}{ Immunosuppression } & \multicolumn{4}{|c|}{ 16s RT-PCR or PCR-ELISA (OspA/B) } \\
\hline & & & & Aorta & Atrium & Ventricle & Apex \\
\hline TO26 & BbN40 & Needle & IC-long term & - & - & nd & - \\
\hline TO29 & & & IC & nd & nd & nd & nd \\
\hline PAX40 & & & IC & nd & nd & nd & nd \\
\hline TO32 & & & IC & - & nd & nd & - \\
\hline E520 & & & IC & + & $+1-$ & nd & - \\
\hline E518 & $\mathrm{BbN} 40$ & Needle & IC-short term & nd & - & nd & nd \\
\hline M14 & & & IC & - & - & - & - \\
\hline M15 & & & IC & - & - & - & - \\
\hline $23 \mathrm{Z}$ & & & IC & nd & + & nd & nd \\
\hline E680 & & & IC & - & - & nd & - \\
\hline 1616 & & & IC & nd & - & nd & nd \\
\hline 652 & $\mathrm{BbN} 40$ & Needle & IS & + & + & + & + \\
\hline 1538 & & & IS & nd & + & nd & + \\
\hline Z1 & & & IS & nd & + & nd & + \\
\hline PAX219 & & & IS & nd & + & nd & + \\
\hline 1614 & & & IS & nd & + & nd & + \\
\hline 30794 & & & IS & + & + & + & + \\
\hline 372 & & & IS & + & + & + & + \\
\hline 099 & $\mathrm{BbN} 40$ & Needle & TISP & nd & nd & + & nd \\
\hline 177 & & & TISP & nd & nd & + & + \\
\hline 199 & & & TISP & + & nd & nd & nd \\
\hline 383 & & & TISP & + & nd & nd & nd \\
\hline 154 & BbN40 & Tick & TISP & + & + & nd & nd \\
\hline 192 & & & TISP & + & + & nd & nd \\
\hline 211 & & & TISP & + & + & + & + \\
\hline 242 & & & TISP & + & nd & nd & nd \\
\hline
\end{tabular}

nd $=$ not determined

Table 5 Spirochetal load ${ }^{\text {a }}$ by Taqman RT-PCR in the heart and aorta of IS NHPs inoculated with $B$. burgdorferi sensu stricto strain $\mathrm{N} 40$

\begin{tabular}{lrrrr}
\hline NHP ID & Aorta & Atrium & Ventricle & \multicolumn{1}{c}{ Apex } \\
\hline 652 & 16100 & 2750 & 83 & 2960 \\
30794 & 11042 & 426095 & 468452 & 836041 \\
372 & 805619 & 196232 & 164402 & 413009 \\
\hline
\end{tabular}

${ }^{\mathrm{a}}$ Number of spirochetes per $100 \mathrm{mg}$ tissue.

of MAC not only in the membranes from cardiac myocytes (Figure 4c) but also in spirochetes (Figure 4e). Detailed examination of the MAC-stained spirochetes suggested that many appeared intact while others appeared mildly damaged or degraded. To investigate whether MAC deposition increased in the heart as a result of infection, we compared MAC deposition by digital image analysis. The results (Figure 5) showed significantly increased MAC in all groups of inoculated NHPs compared with uninfected controls. The three highest values were for TISP-needle, TISP-tick and IS-BbN40-inoculated NHPs. Mild but significantly increased MAC deposition was also found in the garinii NHPs compared with uninfected controls.

To confirm whether MAC was being deposited in spirochetes as suggested by immunohistochemistry, we did double immunofluorescence staining. The results showed colocalization of MAC and Borrelia proteins on spirochetes (Figure 4b, d, f). Examination of heart sections from IS-NHPs revealed that there were both MAC-positive and MAC-negative spirochetes throughout. These results showed that in steroid-treated NHPs heavily infected with BbN40 MAC binds to but does not kill spirochetes.

\section{B-Lymphocyte Chemoattractant (BLC)}

The previous experiments demonstrated extensive accumulation of plasma cells and antibody in the hearts of NHPs with Lyme carditis. To investigate whether specific B-cell chemokines were being produced in the hearts as a result of the infection that could be responsible for plasma cell infiltration, we looked for the presence of the B-cell chemokine BLC/CXCL13. Digital image analysis of formalinfixed immunostained sections showed significant accumulation of BLC/CXCL13 mainly in IS and 

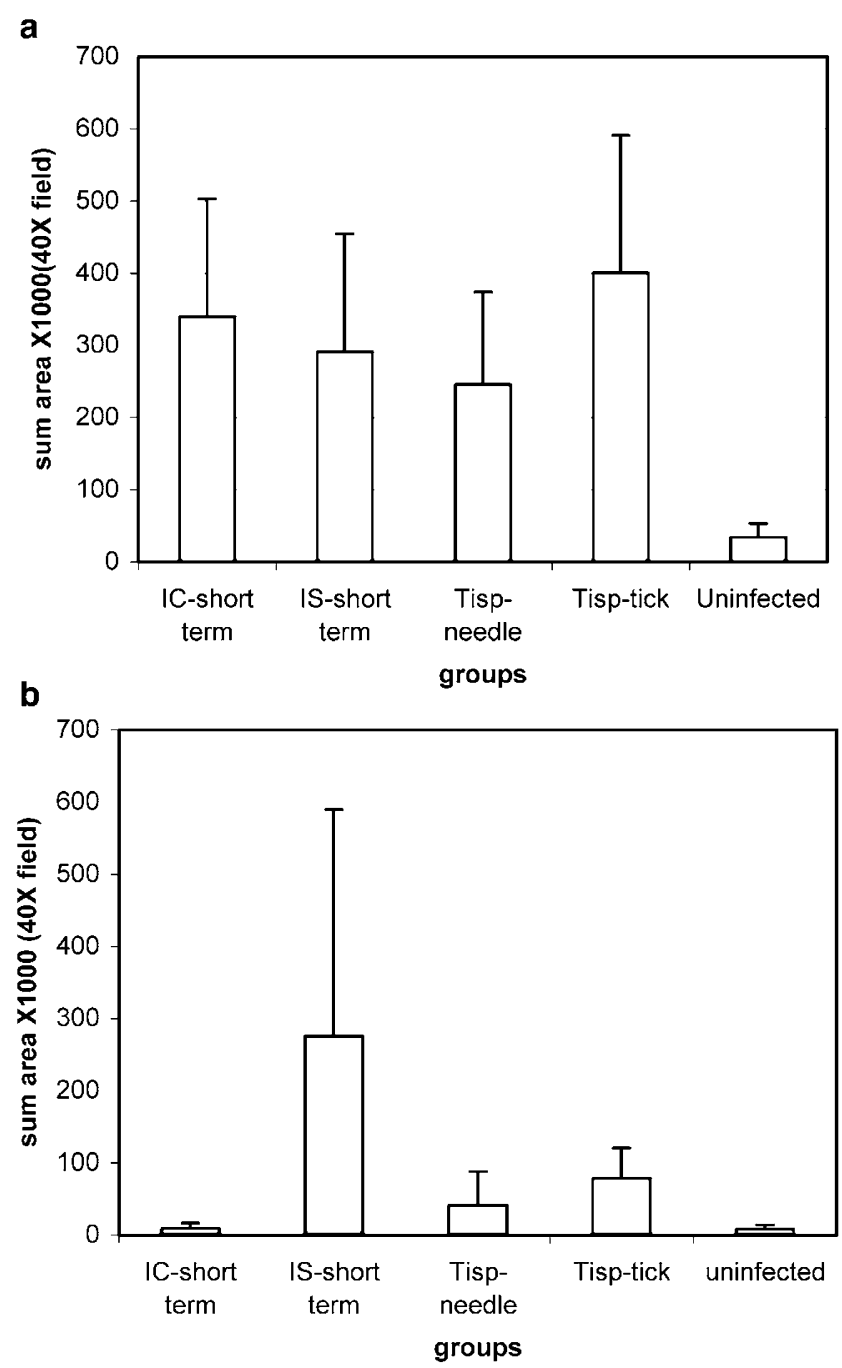

Figure 3 Digital image analysis of immunostaining with antibody anti-IgG (a) or anti-IgM (b) on heart from different groups of NHPs inoculated with $B$. burgdorferi by needle or tick-bite.

Table 6 IgG, IgM and C1q protein levels by quantitative dot blot in the ventricle of NHPs inoculated with BbN40

\begin{tabular}{lccc}
\hline Group & $\operatorname{Ig} G$ & $\operatorname{IgM}$ & $C 1 q$ \\
\hline IS & $2.92 \pm 0.24^{\mathrm{a}}$ & $48.42 \pm 3.49^{\mathrm{b}}$ & $1.16 \pm 0.02^{\mathrm{b}}$ \\
TISP-needle & $3.66 \pm 0.25^{\mathrm{d}}$ & $43.26 \pm 4.19^{\mathrm{c}}$ & $0.57 \pm 0.04^{\mathrm{c}}$ \\
TISP-tick & $3.01 \pm 0.14$ & $36.65 \pm 6.34$ & $0.52 \pm 0.04$
\end{tabular}

${ }^{\mathrm{a}}$ Mean (SD) density per $0.02 \mu \mathrm{g}$ (IgG), $0.2 \mu \mathrm{g}$ (IgM, C1q) of protein extract $\left(\times 1 / 10^{6}\right)$.

${ }^{\mathrm{b}}$ The $P$-value was $<0.001$ for group IS vs TISP.

${ }^{\mathrm{C}}$ The $P$-value was $<0.001$ for group TISP-needle vs TISP-tick.

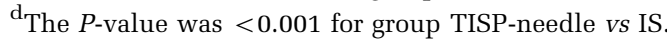

to a lesser extent in IC-short-term and TISP-NHPs (Figure 6). To make sure the signal from the antiBLC/CXCL13 antibody was specific, we repeated the immunostaining with and without blocking with
BLC peptide. The results (not shown) confirmed the anti-BLC antibody was specific.

\section{Discussion}

This manuscript presents the first comprehensive investigation of cardiac involvement in Lyme borreliosis in primates. The main findings of the study were as follows: (1) carditis is very common in NHPs infected with $B$. burgdorferi but is mild unless the animals are immunosuppressed. (2) The spirochetal load in the heart is very high in NHPs necropsied while immunosuppressed but decreased to minimal or undetectable in all NHPs necropsied while immunocompetent. (3) The cellular inflammatory response to the infection was characterized by multifocal collections of $\mathrm{T}$ cells, plasma cells, and macrophages. (4) Infection resulted in increased deposition of IgG and IgM in the heart. (5) Expression of the B-cell chemokine BLC was increased accordingly to the spirochetal load. (6) Increased deposition of the complement membrane attack complex (MAC) was found in the heart from TISP and IS-NHPs, and a significant percentage of the spirochetes in the heart had MAC on their membranes.

A previous report from our group described significant cardiac inflammation and tissue injury in the heart of immunosuppressed NHPs infected with the BbN40 strain. ${ }^{6}$ We had also seen extensive cardiac injury in the heart of mice with severe combined immunodeficiency infected with $B$. turicatae. ${ }^{18}$ In humans with Lyme disease, carditis is found in up to $25 \%$, but only rarely pathology specimens are available for examination. ${ }^{19}$ In NHPs we found that all but one inoculated with the BbN40 strain had evidence of carditis at necropsy, including some that had been inoculated years before. However, carditis was overall mild. The pattern of inflammation was multifocal and patchy, although occasional large lesions were found (color Figure 1a).

All groups of BbN40-inoculated NHPs had similar macrophage infiltration with the exception of the long-term-IC group (Figure 2). The finding of increased numbers of T cells, plasma cells (Table 3, Figure 1c and d), and macrophages (Figure 2) in the heart is consistent with previous observations in small animal models of Lyme carditis. ${ }^{20-22}$ Plasma cells were more abundant in IS-NHPs (Table 3), which was also the group with the highest expression of BLC (Figure 6) and the highest tissue deposition of IgM (Figure 4, panel b). This suggests that one of the consequences of persistent $B$. burgdorferi infection of the heart is upregulation of the B-cell chemokine BLC leading to infiltration by plasma cells and production and deposition of large amounts of IgM. The specificity of the IgM antibody deposited in heart tissue has not been determined. It is also unknown why this IgM antibody is unable to 

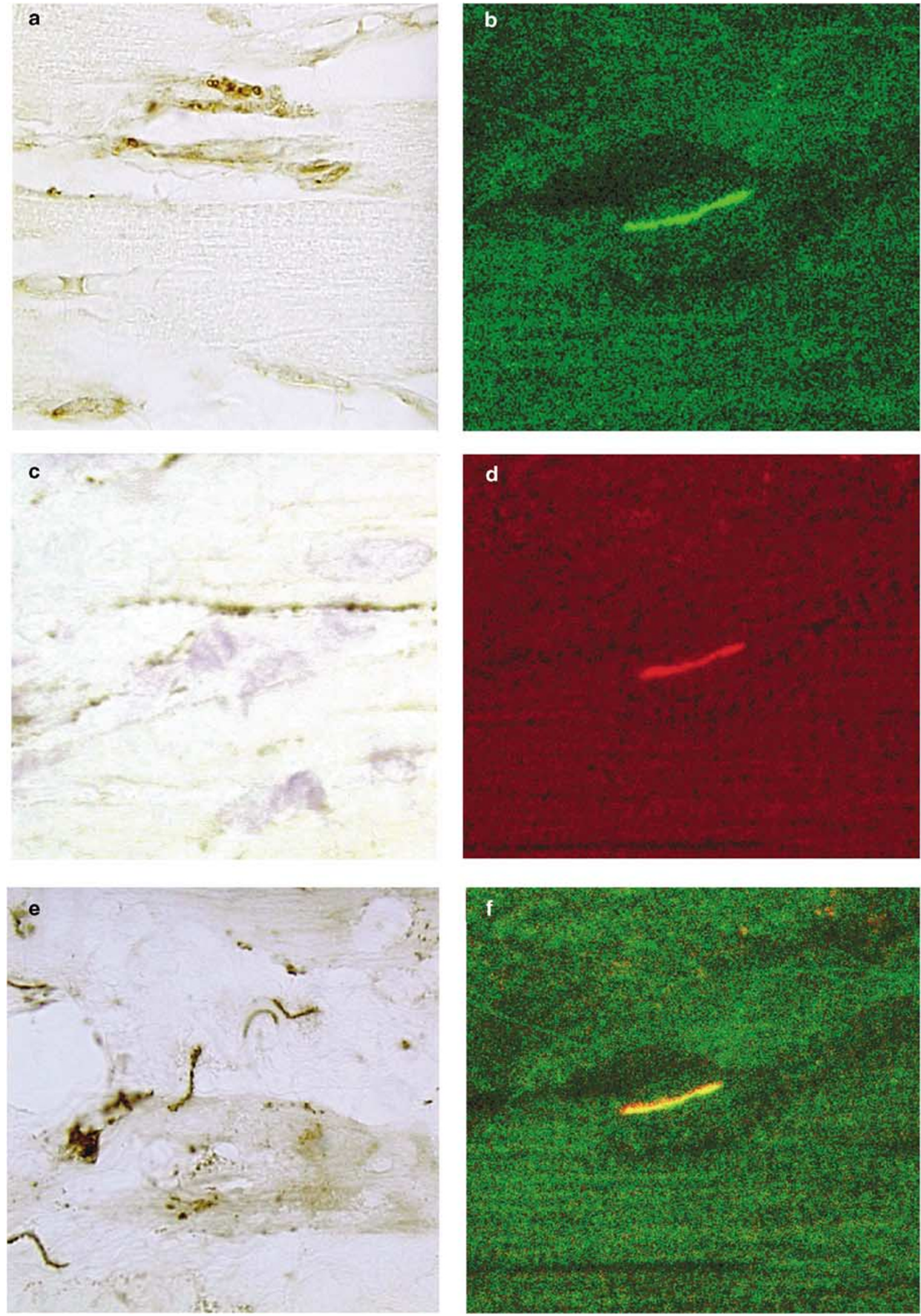

Figure $4 \mathrm{C} 1 \mathrm{q}$ (panel a) and MAC (panels $\mathbf{c}$ and e) immunostaining in the heart of IS NHP 31372. MAC \& Borrelia double immunofluorescent staining with FITC conjugate anti BbN40 antibody (panel b, green color), TRITC conjugated anti MAC antibody (panel $\mathbf{d}$, red color) and merged images from panels $\mathbf{b}$ and $\mathbf{d}$ (panel f, yellow color). 


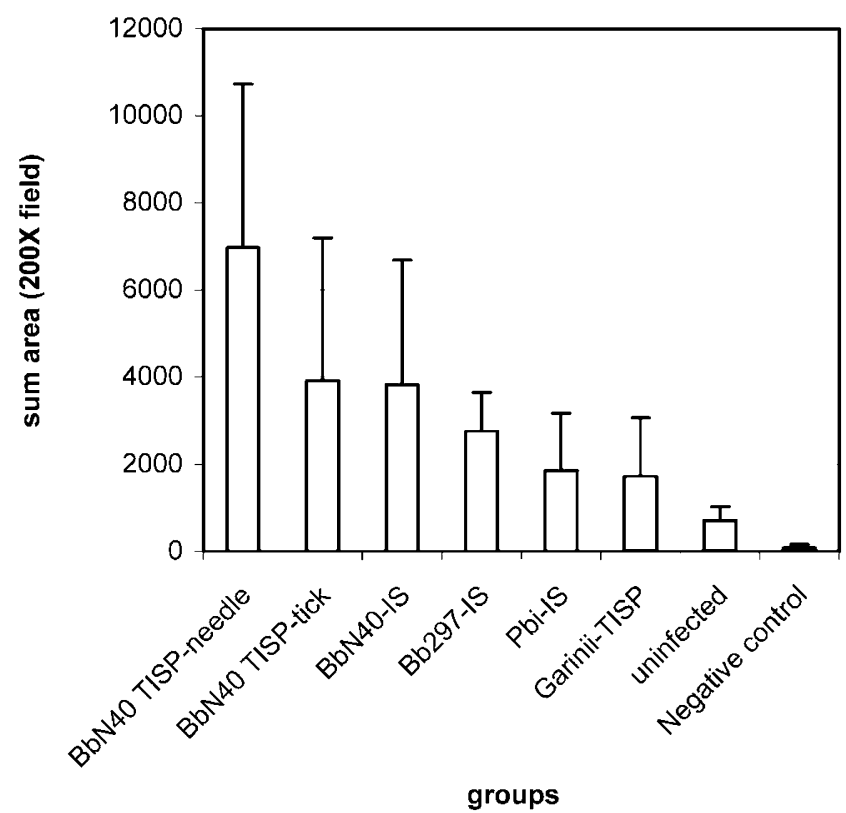

Figure 5 Digital image analysis of immunostaining with antibody anti-MAC (C9 neoepitope) on heart from different groups of NHPs inoculated with different genospecies of $B$. burgdorferi.

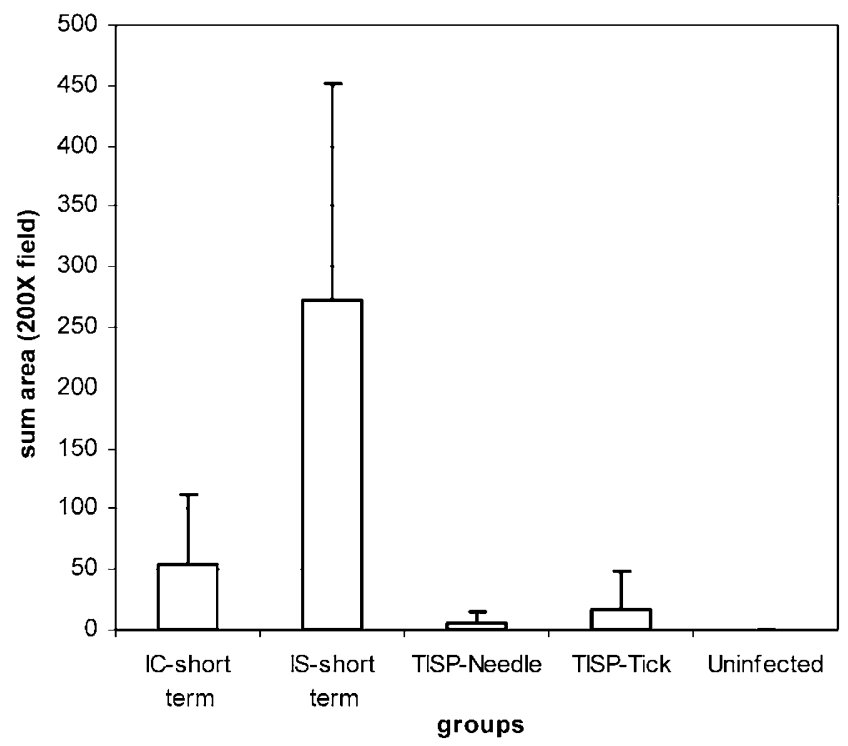

Figure 6 Digital image analysis of immunostaining with antibody anti-BLC on heart from different groups of NHPs inoculated with $\mathrm{BbN} 40$.

kill the spirochetes, as shown by the very high spirochetal load present in the heart of IS-NHPs (Table 5).

BLC (also called BCA-1 or CXCL13) is a chemokine thought to be especially selective for B-cells. BLC is considered a homing chemokine and has been implicated in the trafficking of lymphocytes and dentritic cells in lymphoid organs, and is critical for lymphoid neogenesis ${ }^{23}$ and for establishment of lymphoid follicle-like areas in chronically inflamed tissues such as salivary glands in Sjogren's syndrome ${ }^{24}$ or joins in rheumatoid arthritis. ${ }^{25}$ In a previous study, we found significantly increased levels of BLC mRNA in skeletal muscle from TISPNHPs inoculated with BbN40 compared with controls that were uninfected or inoculated but not infected. ${ }^{26}$

As expected, a dramatic effect of the immunosuppression was an inability to fight the infection. One reason why IS-NHPs failed to control the infection was the lower levels of circulating specific antibody, as previously reported..$^{5,7}$ Consistent with this are the results of the dot-blot analysis (Table 6) that showed higher total IgG in the heart of TISP compared with IS-NHPs. Although digital image analysis showed similar levels of IgG, this is a less sensitive technique than the dot blot. In contrast to IgG, IS-NHPs had much higher IgM as shown both by dot blot and digital image analysis. As discussed above, it is unclear why this IgM antibody failed to control the infection.

Another possibility for the higher spirochetal load in steroid-treated animals is impaired complement activation that is required for efficient spirochetal killing. In the absence of specific antibody $B$. burgdorferi is resistant to the bactericidal activity of complement. ${ }^{27}$ Bactericidal antibody appears necessary for the effective formation of MAC. ${ }^{27}$ Most pathogenic microorganisms, and in particular those that circulate in the blood stream like spirochetes, develop a wide range of strategies to elute antibody and complement killing. We found that a large percentage of spirochetes in the heart of IS-NHPs had MAC on their membranes (color Figure 4e), including many that appear morphologically intact. The reason why spirochetes appear to survive MAC deposition in IS-NHPs is not known. One possibility is disabling the correct assembly of MAC. MAC is an ultrastructurally heterogenous complex that induces the formation of membrane channels of different sizes. ${ }^{28}$ Patarakul et $a{ }^{29}$ found similar level of MAC on the membrane of a complement-resistant $B$. burgdorferi (WT297) and a complement-sensitive mutant (MUT297). Although both had polymerization of C9 and MAC diffusely distributed and tightly bound on the outer membrane, protease treatment rendered WT297 but not MUT297 susceptible to serum killing. Proteins of 20,30, and $66 \mathrm{kDa}$ were found in the membrane of WT297 but not in MUT297 that may be responsible for complement resistance. ${ }^{29}$ Two type of proteins that may be involved in complement resistance in $B$. burgdorferi have been reported. One of them is OspE/Erp proteins that bind factor $\mathrm{H}^{30,31}$ More recently, a CD59-like molecule that inhibits the assembly of MAC was described on the outer membrane of B. burgdorferi. ${ }^{32}$

We found deposition of MAC in the heart of IS-NHPs not only on spirochetes but also on the membranes of cardiac myocytes. In Chagas cardiomyopathy, another form of infectious carditis caused by parasite Trypanosoma cruzi, MAC was found in 
the sarcolemma of $38 \%$ of cases compared with $0 \%$ of controls. ${ }^{33}$ MAC deposition is also a feature of damaged myocytes in myocardial infarction. ${ }^{34} \mathrm{We}$ propose that in Lyme carditis complement activation in response to the spirochetal infection leads to MAC deposition in cell membranes of cardiac myositis with secondary fiber degeneration and fibrosis.

The true prevalence of Lyme carditis in humans is difficult to determine because only few cases have been examined at autopsy. The incidence of symptomatic Lyme carditis has been estimated to be 4$10 \%$ in adults. However, the incidence of abnormal ECG findings in asymptomatic patients with probable or definite Lyme borreliosis is higher, $29 \%$ in one study in children. ${ }^{35}$ The clinical course of Lyme carditis is usually benign with most patients recovering completely. In rare instances, death has been reported. ${ }^{19,36}$ The cardinal manifestation is conduction system disease, which generally is selflimited. Heart block occurs usually at the level of the atrioventricular node but often is unresponsive to atropine sulfate. Temporary pacing may be necessary in more than $30 \%$ of patients, but permanent heart block rarely develops. Myocardial and pericardial involvement can occur but generally is mild and self-limited. ${ }^{37}$ Cardiomyopathy has been associated with $B$. burgdorferi infection in Europe but not in the United States. ${ }^{4}$ No treatment has been shown clearly to attenuate or prevent the development of Lyme carditis, but mild carditis generally is treated with oral antibiotics and severe carditis with intravenous antibiotics. ${ }^{37}$

Studies in mouse models of Lyme borreliosis showed that Lyme carditis is very frequent but until now the true incidence in primates was not known. Histopathological examination of mice with severe combined $(\text { scid })^{38}$ or other $(\mathrm{NIH}-3)^{39}$ immunodeficiency inoculated with $B$. burgdorferi show high prevalence of severe carditis. Lyme carditis is also prominent in immunocompetent mice $^{20,21}$ and is worse in IL-4-deficient mice. ${ }^{22}$ In $\mathrm{C} 3 \mathrm{H}$ mice, spirochetes have been found in the heart as early as 6 days after inoculation and all mice of the $\mathrm{C} 3 \mathrm{H}$ and C57BL/6 haplotypes had infected hearts. ${ }^{20}$ The spirochetes had a predilection for connective tissue in the heart base. Carditis was first detectable on day 10 , peaked on day 15, and resolved except for persistence of periaortic lymphoplasmacytic infiltrates in all mice. The $\mathrm{C} 3 \mathrm{H}$ mice developed more severe disease than the C57BL/6 mice, and this was associated with earlier appearance, greater numbers, and later clearance of spirochetes in $\mathrm{C} 3 \mathrm{H}$ mice. ${ }^{20} \mathrm{~A}$ previous study of rhesus macaques inoculated with the JD-1 strain of $B$. burgdorferi found focal myocarditis in three out of six hearts examined at necropsy 6 months later. ${ }^{40}$

In summary, this study revealed Lyme carditis is very common in infected NHPs when examined pathologically. Although severe infection of the heart occurs in the setting of immunosuppression, the intact immune response of NHPs reduced the infection to minimal or undetectable levels. The failure of steroid-treated NHPs to clear the infection may be the result of impaired killing due to decreased production of specific antibody or failure of MAC assembly on the membranes of spirochetes.

\section{Acknowledgements}

This work was supported by Contract DMID-99-03 from the NIH-NIAID. Dr Cadavid is a recipient of a Scientist Development Grant from the American Heart Association-Heritage Affiliate. We thank Dr Bettina Wilske (Max Von Pettenkofer Institut fur Hygiene und Medizinische Mikrobiologie, Munich, Germany) and Dr Martin Schriefer (CDC, Fort Collins, Colorado) for providing the $B$. garinii isolates. The assistance of the staff at the California Primate Research Center is greatly appreciated.

\section{References}

1 Steere AC. Lyme disease. N Engl J Med 2001;345: 115-125 (see comments).

2 Lyme disease-United States, 2000. Morb Mortal Wkly Rep 2002;51:29-31.

3 Nagi KS, Joshi R, Thakur RK. Cardiac manifestations of Lyme disease: a review. Can J Cardiol 1996;12:503-506.

4 Haddad FA, Nadelman RB. Lyme disease and the heart. Front Biosci 2003;8:s769-s782.

5 Bai Y, Narayan K, Dail D, et al. Spinal cord involvement in the nonhuman primate model of Lyme disease. Lab Invest 2004;84:160-172.

6 Cadavid D, O’Neill T, Schaefer H, Pachner AR. Localization of Borrelia burgdorferi in the nervous system and other organs in a nonhuman primate model of lyme disease. Lab Invest 2000;80:1043-1054.

7 Cadavid D, Bai Y, Dail D, et al. Infection and inflammation in skeletal muscle from nonhuman primates infected with different genospecies of the Lyme disease spirochete Borrelia burgdorferi. Infect Immun 2003;71:7087-7098.

8 Pachner AR, Delaney E, O’Neill T. Neuroborreliosis in the nonhuman primate: Borrelia burgdorferi persists in the central nervous system. Ann Neurol 1995;38: 667-669.

9 Pachner AR, Delaney E, O’Neill T, et al. Inoculation of nonhuman primates with the N40 strain of Borrelia burgdorferi leads to a model of Lyme neuroborreliosis faithful to the human disease. Neurology 1995;45: 165-172.

10 Pachner AR, Schaefer H, Amemiya K, et al. Pathogenesis of neuroborreliosis-lessons from a monkey model. Wien Klin Wochenschr 1998;110:870-873.

11 Moody KD, Barthold SW, Terwilliger GA. Lyme borreliosis in laboratory animals: effect of host species and in vitro passage of Borrelia burgdorferi. Am J Trop Med Hyg 1990;43:87-92.

12 Masuzawa T, Beppu Y, Kawabata H, et al. Experimental Borrelia burgdorferi infection of outbred mice. J Clin Microbiol 1992;30:3016-3018. 
13 Busch U, Hizo-Teufel C, Boehmer R, et al. Three species of Borrelia burgdorferi sensu lato (B. burgdorferi sensu stricto, B. afzelii, and B. garinii) identified from cerebrospinal fluid isolates by pulsed-field gel electrophoresis and PCR. J Clin Microbiol 1996;34: 1072-1078.

14 Pachner AR, Delaney E, Zhang WF, et al. Protection from Lyme neuroborreliosis in nonhuman primates with a multiantigenic vaccine. Clin Immunol 1999;91: 310-313.

15 Pachner AR, Braswell ST, Delaney E, et al. A rabbit model of Lyme neuroborreliosis: characterization by PCR, serology, and sequencing of the OspA gene from the brain. Neurology 1994;44:1938-1943.

16 Pachner AR, Cadavid D, Shu G, et al. Central and peripheral nervous system infection, immunity, and inflammation in the NHP model of Lyme borreliosis. Ann Neurol 2001;50:330-338.

17 Pachner AR, Amemiya K, Bartlett $\mathrm{M}$, et al. Lyme borreliosis in rhesus macaques: effects of corticosteroids on spirochetal load and isotype switching of antiBorrelia burgdorferi antibody. Clin Diagn Lab Immunol 2001;8:225-232.

18 Cadavid D, Thomas DD, Crawley R, et al. Variability of a bacterial surface protein and disease expression in a possible mouse model of systemic Lyme borreliosis. J Exp Med 1994;179:631-642.

19 Cary NR, Fox B, Wright DJ, et al. Fatal Lyme carditis and endodermal heterotopia of the atrioventricular node. Postgrad Med J 1990;66:134-136.

20 Armstrong AL, Barthold SW, Persing DH, et al. Carditis in Lyme disease susceptible and resistant strains of laboratory mice infected with Borrelia burgdorferi. Am J Trop Med Hyg 1992;47:249-258.

21 Ruderman EM, Kerr JS, Telford III SR, et al. Early murine Lyme carditis has a macrophage predominance and is independent of major histocompatibility complex class II-CD4+ T cell interactions. J Infect Dis 1995; 171:362-370.

22 Satoskar AR, Elizondo J, Monteforte GM, et al. Interleukin-4-deficient BALB/c mice develop an enhanced Th1-like response but control cardiac inflammation following Borrelia burgdorferi infection. FEMS Microbiol Lett 2000;183:319-325.

23 Ruddle NH. Lymphoid neo-organogenesis: lymphotoxin's role in inflammation and development. Immunol Res 1999;19:119-125.

24 Amft N, Curnow SJ, Scheel-Toellner D, et al. Ectopic expression of the B cell-attracting chemokine BCA-1 (CXCL13) on endothelial cells and within lymphoid follicles contributes to the establishment of germinal center-like structures in Sjogren's syndrome. Arthritis Rheum 2001;44:2633-2641.

25 Takemura S, Braun A, Crowson C, et al. Lymphoid neogenesis in rheumatoid synovitis. J Immunol 2001; 167:1072-1080.
26 Pachner AR, Dail D, Narayan K, et al. Increased expression of B-lymphocyte chemoattractant, but not pro-inflammatory cytokines, in muscle tissue in rhesus chronic Lyme borreliosis. Cytokine 2002;19:297-307.

27 Kochi SK, Johnson RC, Dalmasso AP. Complementmediated killing of the Lyme disease spirochete Borrelia burgdorferi. Role of antibody in formation of an effective membrane attack complex. J Immunol 1991;146:3964-3970.

28 Tschopp J. Ultrastructure of the membrane attack complex of complement. Heterogeneity of the complex caused by different degree of C9 polymerization. J Biol Chem 1984;259:7857-7863.

29 Patarakul K, Cole MF, Hughes CA. Complement resistance in Borrelia burgdorferi strain 297: outer membrane proteins prevent MAC formation at lysis susceptible sites. Microb Pathogen 1999;27:25-41.

30 Hellwage J, Meri T, Heikkila T, et al. The complement regulator factor $\mathrm{H}$ binds to the surface protein OspE of Borrelia burgdorferi. J Biol Chem 2001;276:8427-8435.

31 Stevenson B, El-Hage N, Hines MA, et al. Differential binding of host complement inhibitor factor $\mathrm{H}$ by Borrelia burgdorferi Erp surface proteins: a possible mechanism underlying the expansive host range of Lyme disease spirochetes. Infect Immun 2002;70: 491-497.

32 Pausa M, Pellis V, Cinco M, et al. Serum-resistant strains of Borrelia burgdorferi evade complementmediated killing by expressing a CD59-like complement inhibitory molecule. J Immunol 2003;170:3214-3222.

33 Aiello VD, Reis MM, Benvenuti LA, et al. A possible role for complement in the pathogenesis of chronic chagasic cardiomyopathy. J Pathol 2002;197:224-229.

34 Yasojima K, Schwab C, McGeer EG, et al. Human heart generates complement proteins that are upregulated and activated after myocardial infarction. Circ Res 1998;83:860-869.

35 Woolf PK, Lorsung EM, Edwards KS, et al. Electrocardiographic findings in children with Lyme disease. Pediatr Emerg Care 1991;7:334-336.

36 Marcus LC, Steere AC, Duray PH, et al. Fatal pancarditis in a patient with coexistent Lyme disease and babesiosis. Demonstration of spirochetes in the myocardium. Ann Intern Med 1985;103:374-376.

37 Pinto DS. Cardiac manifestations of Lyme disease. Med Clin North Am 2002;86:285-296.

38 Schaible UE, Kramer MD, Justus CW, et al. Demonstration of antigen-specific $\mathrm{T}$ cells and histopathological alterations in mice experimentally inoculated with Borrelia burgdorferi. Infect Immun 1989;57:41-47.

39 Defosse DL, Duray PH, Johnson RC. The NIH-3 immunodeficient mouse is a model for Lyme borreliosis myositis and carditis. Am J Pathol 1992;141:3-10.

40 Roberts ED, Bohm Jr RP, Cogswell FB, et al. Chronic lyme disease in the rhesus monkey. Lab Invest 1995; 72:146-160. 